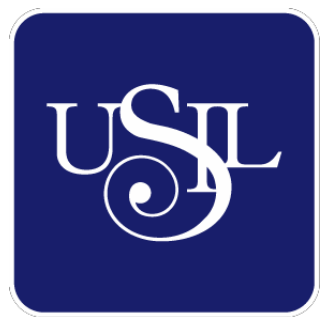

UNIVERSIDAD

SAN IGNACIO

DE LOYOLA

ESCUELA DE POSTGRADO

Doctorado en Nutrición y Alimentos

\title{
RELACIÓN ENTRE LA DIABETES GESTACIONAL Y EL SÍNDROME METABOLICO EN EL INSTITUTO MATERNO PERINATAL, LIMA, 2018
}

Tesis para optar el Grado de Doctor en Nutrición y Alimentos

CECIBEL GUISELA APARCANA ESPINOZA

\author{
Asesor \\ Dr. Carlos Alvarado Ortiz Ureta \\ Lima - Perú \\ 2019
}


Dedicatoria

Esta tesis está dedicada a Dios porque ha estado conmigo a cada paso que doy, cuidándome y dándome fortaleza para continuar; A mis padres, por ser el pilar fundamental en todo lo que soy, en toda mi educación, tanto académica, como de la vida, por su incondicional apoyo perfectamente mantenido a través del tiempo quienes a lo largo de mi vida han velado por mi bienestar y educación siendo mi apoyo en todo momento. A mi esposo e hijo por haber depositado su entera confianza en cada reto que se me presentaba sin dudar ni un solo momento en mi inteligencia y capacidad. 


\section{Agradecimiento}

Mi profundo agradecimiento a todas las autoridades y personal de la Universidad San Ignacio Loyola de manera especial a al Dr. Carlos Alvarado, a la Dra. Teresa Blanco, al Dr. Felipe Conchoy y la Dra. Ana María Muñoz quienes con la enseñanza de sus valiosos conocimientos hicieron que pueda crecer aún más cada día a día como profesional, gracias a cada una de ustedes

por su paciencia, dedicación, apoyo incondicional y amistad. 
Objetivo: determinar la relación entre la diabetes gestacional y el síndrome metabólico en el Instituto Materno Perinatal, Lima, 2018. Materiales y métodos: Se realizó un estudio observacional, analítico de corte transversal, tipo caso-control. Participaron 412 gestantes y se seleccionaron una muestra aleatoria en dos grupos gestantes siendo 206 casos con diagnóstico de diabetes gestacional y 206 controles gestantes sin diagnóstico de diabetes gestacional del consultorio de Nutrición del Instituto Nacional Materno Perinatal. El síndrome metabólico se midió según la National Cholesterol Education Program-Adult Treatment Panel III). Resultados: Existe mayor prevalencia en gestantes de 20 a 35 años de edad $88.8 \%$ casos y $93.2 \%$ control y de acuerdo al tiempo gestacional de 20 a 40 semanas con 95,6\% casos y $96.1 \%$ control. La diabetes gestacional se presentó en un $68,9 \%$ y el 7,8\% en los controles. La prevalencia del síndrome metabólico en las gestantes fue el $100 \%$ en los casos y el 7,8\% en los controles. Los criterios del síndrome metabólico en los casos, la mayor prevalencia se presentó con HTA $(95,6 \%)$, seguido de la obesidad abdominal pregestacional con el $86,4 \%$, la diabetes mellitus en el 68,9\% y la hipertrigliceridemia con el 40,7\% y el 24,7\% de los estudiados presentó el colesterol HDL bajo. En el grupo control se observó al 7,8\% con HTA, diabetes mellitus y obesidad abdominal pregestacional, respectivamente. Conclusiones: Existe una relación significativa entre la diabetes gestacional y el síndrome metabólico en la gestante con un Rango de Prevalencia 7.69 y una significancia de 0,000. Siendo que las gestantes con diabetes gestacional aumentan 8 veces el riesgo de padecer síndrome metabólico.

Palabras clave: diabetes gestacional, síndrome metabólico, gestante.

\section{ABSTRACT}




\section{Summary:}

Objective: Establish the relationship between gestational diabetes and the metabolic syndrome at the Instituto Materno Perinatal (Maternal Perinatal Institute), Lima, 2018. Materials and methods: It has been made an observational study analytical of crosssection sort case-control. There were 412 pregnant women and there were selected an aleatory sample in two pregnant groups being 206 cases with gestational diabetes diagnosis and 206 pregnant controls without gestational diabetes diagnosis from the nutrition office of the Instituto Nacional Materno Perinatal (National Institute Maternal Perinatal) The metabolic syndrome was measured according to the National Cholesterol Education Program-Adult Treatment Panel III). Results: There is a higher prevalence in pregnant women from 20 to 35 years old $88,8 \%$ cases and 93,2\% control and according to the gestational time from 20 to 40 weeks with $95,6 \%$ cases and $96,1 \%$ control. Gestational diabetes was presented in $68,9 \%$ and the $7,8 \%$ of the controls. The prevalence of the metabolic syndrome in the pregnant women was the $100 \%$ of the cases and the $7,8 \%$ of the controls. The criterions of the metabolic syndrome in the cases, the higher prevalence was presented with A.H(arterial hypertension, 95,6\%) followed by the pregestational abdominal obesity with $86,4 \%$, diabetes mellitus in $68,9 \%$ and the hypertriglyceridemia with $40,7 \%$ and $24,7 \%$ of the studied had low HDL cholesterol. In the control group was observed the $7,8 \%$ with A.H(arterial hypertension), diabetes mellitus and pregestational abdominal obesity respectively. Conclusions: There is a significant relationship between the gestational diabetes and the metabolic syndrome in the pregnant woman with a range of prevalence 7,69 and a significance of 0,000 . So the pregnant woman with gestational diabetes increase 8 times the risk of suffer metabolic syndrome.

Keywords: gestational diabetes, metabolic syndrome, pregnant. 


\section{ÍNDICE}

Introducción

CAPÍTULO I

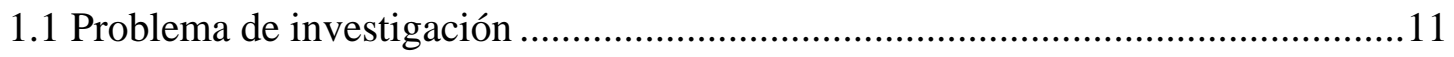

1.1.1 Planteamiento del problema ................................................................. 11

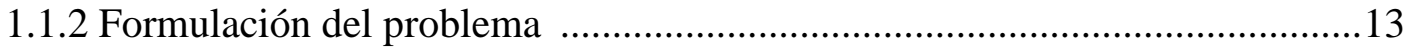

1.2 Justificación de la investigación ......................................................................... 14

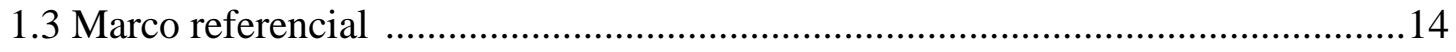

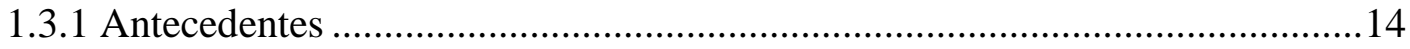

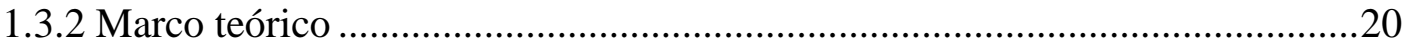

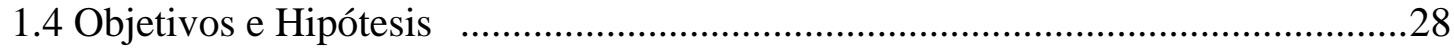

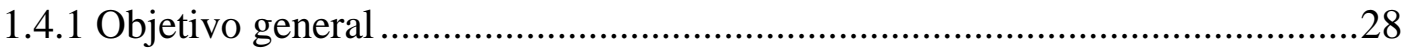

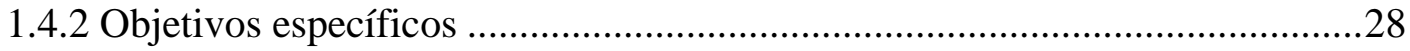

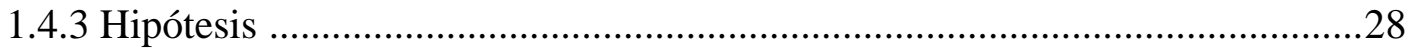

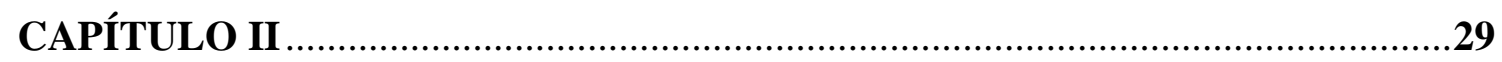

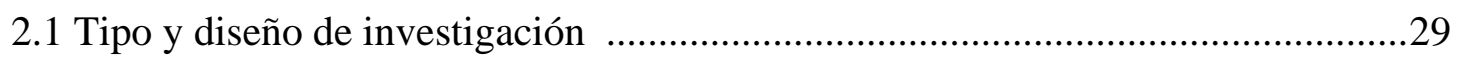

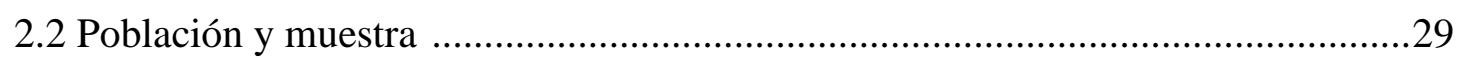

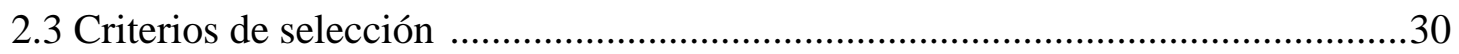

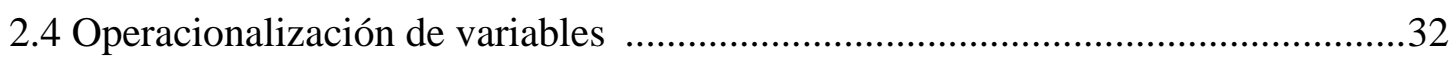

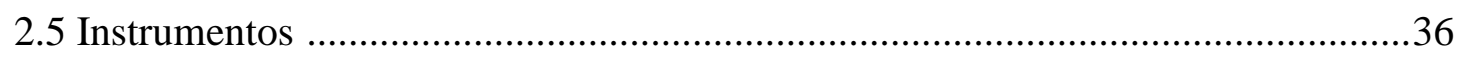

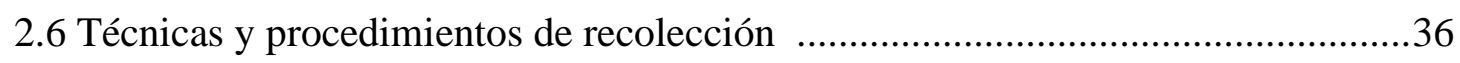

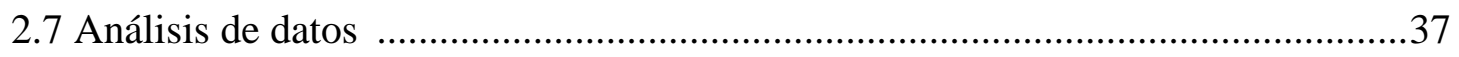

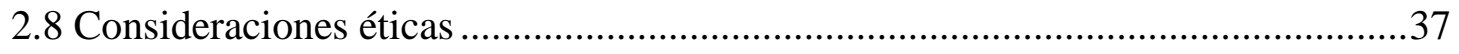

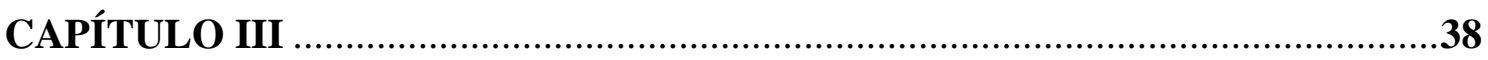

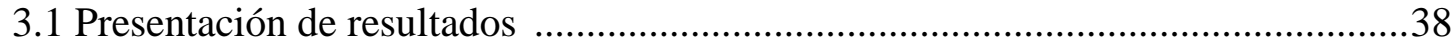

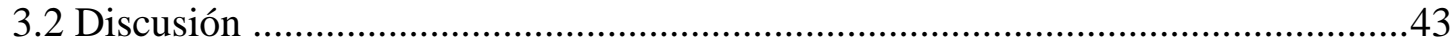

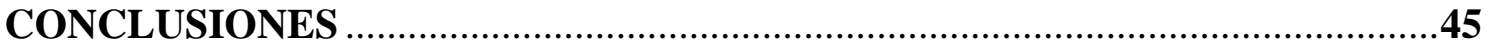

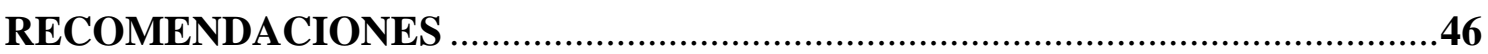




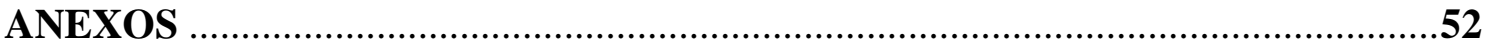

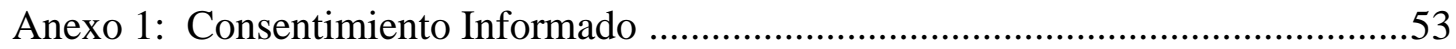

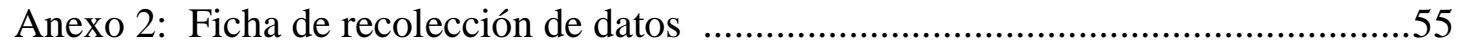

Anexo 3: Prueba binomial de jueces expertos .......................................................56

Anexo 4: Prueba de validez del instrumento ............................................................57

Anexo 5: Prueba de confiabilidad del instrumento ...................................................58 


\section{Introducción}

El embarazo es un periodo en la que se puede presentar una resistencia a la insulina, causado por el estrés en las células beta en el páncreas. Además, el incremento de las hormonas en este periodo es muy frecuente conllevando a la disminución de la glucosa en ayunas, también en los almacenes grasos y un incremento del apetito.

Conforme el periodo gestacional avanza, es menor la sensibilidad a la insulina, por ende, los valores de glucosa en la gestante aumentan para ofrecerla al feto. Esta transferencia de energía entre el binomio madre - feto proporciona valores de glucosa cerca de los 70 minutos postprandiales de $120 \mathrm{mg} / \mathrm{dl}$.

Una de las complicaciones en la embarazada es la diabetes mellitus gestacional (DMG), la cual se presenta cuando la insulina secretada no cubre la sensibilidad de esta; esta enfermedad.

La diabetes mellitus (DM) es considerada en nuestro país como un "problema de salud pública" y es una de las principales enfermedades no transmisibles (ENT) cuya prevalencia de diabetes se ha incrementado progresivamente en los últimos años.

La DM es una de las enfermedades que se incluyen en la definición del síndrome metabólico (SM), en la cual se presentan varios problemas de salud con una aparición de forma simultánea o secuencial en la persona, con un origen en factores genéticos y ambientales que combinados al estilo de vida, pueden desencadenar el SM, destacando que la resistencia a la insulina sería el componente causal. 
El SM en su definición está caracterizado por cinco criterios para su diagnóstico, siendo el principal la obesidad abdominal, los triglicéridos séricos elevados, el colesterol HDL bajo, hipertensión arterial (HTA) y los niveles elevados de glicemia.

Diversos estudios a nivel mundial han encontrado que los factores de riesgo asociados al SM son la resistencia a la insulina, que causa la diabetes tipo 2, la HTA y la enfermedad cardiovascular.

La presente tesis doctoral tiene la finalidad en determinar la relación entre la diabetes gestacional y el síndrome metabólico en el Instituto Materno Perinatal, Lima, 2018. 


\section{CAPÍTULO I}

\subsection{Problema de investigación}

\subsubsection{Planteamiento del problema}

A nivel mundial las personas con Diabetes Mellitus (DM) han aumentado cada año, así encontramos que en los años 80 la prevalencia era de 108 millones y en la actualidad son 422 millones según la Organización Mundial de la Salud (OMS); con mayor énfasis en los países de mayor pobreza. ${ }^{1} \mathrm{La}$ DM trae consigo repercusiones negativas en la salud del individuo como ceguera, complicaciones en el riñón, infarto de miocardio, accidente cerebrovascular y amputación en los miembros inferiores. Llevando a la mortalidad a quienes la padecen con una tasa de 1,8 millones de muertes. ${ }^{1}$

Los reportes de la OMS revelan que la DM para el año 2030 será la séptima causa de muerte en el mundo. ${ }^{1}$ La prevención y/o retraso de la DM tipo 2 es posible mediante el abordaje en el consumo de una alimentación saludable, la práctica de actividad física, contar con un peso adecuado y la restricción en el consumo de tabaco. $^{1}$

Se puede tratar la diabetes y evitar o retrasar sus consecuencias con dieta, actividad física, medicación y exámenes periódicos para detectar y tratar sus complicaciones. ${ }^{1}$ La prevalencia de la diabetes gestacional se presenta entre el 1 al $14 \%$ a nivel mundial. ${ }^{2}$ En el Perú, la diabetes mellitus gestacional se encuentra en el $16 \%^{3}$ de las embarazadas. Estos valores siguen creciendo y se asocian con 
efectos maternos y en el feto de forma negativa. "Las mujeres con diabetes mellitus gestacional tienen mayor riesgo de padecer diabetes tipo 2 en un futuro cercano a su embarazo y sus hijos tienen mayor riesgo de padecer obesidad y diabetes a largo plazo".2

Los reportes de la prevalencia de la DM en la gestación muestran no solo la influencia de la genética y las causas ambientales, por otro lado la falta de unificación en el diagnóstico y las estrategias en el abordaje a esta patología. ${ }^{4}$ La diabetes gestacional es un tema de mucha controversia en el mundo no solo por su definición, también por los protocolos de cribado, criterios diagnósticos y estrategias de tratamiento. ${ }^{4}$

El tratamiento exitoso en la gestante con diabetes se fundamenta en el control de las pacientes que son diabéticas pregestacionales, aunque hay factores que desencadenan la DG recién durante el embarazo. ${ }^{5}$ Los factores de riesgo asociados con la diabetes mellitus gestacional son: "el sobrepeso y la obesidad, el número de embarazos, ganancia de peso materno mayor de $20 \mathrm{~kg}$ durante el embarazo, antecedentes de complicaciones obstétricas, edad materna, antecedente de DM, glucosuria, macrosomía y abortos previos". ${ }^{6}$

La diabetes mellitus gestacional "se define como cualquier grado de intolerancia a la glucosa, que es reconocido o identificado por primera vez en el embarazo". 6 La morbimortalidad fetal, asociada con la macrosomía es una de las mayores complicaciones en la diabetes mellitus gestacional. Diversas investigaciones han concluido que la DG es un factor predictivo de DM y el riesgo cardiovascular posterior al parto. ${ }^{2}$ 
Por otro lado, en mujeres diagnosticas con DM sus hijos presentaran un mayor riesgo de padecer macrosomía como con el bajo peso al nacimiento. ${ }^{7}$ Una explicación con origen genético revela que la exposición a un medio metabólico anómalo durante el desarrollo intrauterino puede ocasionar una disfunción de las células beta y/o alteraciones en los hepatocitos y adipocitos, que se pueden presentar desde la niñez hasta la etapa adulta. ${ }^{1}$

Así como se presentan una serie de alteraciones asociadas a la DM como la obesidad, dislipidemia, HTA, resistencia insulínica definidas como síndrome metabólico, esta condición no es ajena en el embarazo y es llamada síndrome metabólico gestacional (SMG). ${ }^{2}$

\subsubsection{Formulación del problema}

¿Cuál es la relación entre la diabetes gestacional y el síndrome metabólico en el Instituto Materno Perinatal, Lima, 2018?

\subsection{Justificación de la investigación}

1.2.1 Conveniencia: La diabetes gestacional es una entidad que genera mayor riesgo feto neonatal, razón por la cual es necesario conocer los factores de riesgo que en la actualidad se presentan mayor frecuencia en los casos de diabetes gestacional.

1.2.2 Relevancia Social: Además de establecer un referente para orientar al personal sanitario de primer nivel de atención para la identificación de pacientes con factores de riesgo de padecer esta enfermedad, así como la realización del diagnóstico y la implementación del tratamiento temprano y oportuno, antes de enviar a las pacientes al segundo y tercer nivel de atención. 
1.2.3 Implicaciones Prácticas: estudiar intervenciones factibles en la práctica clínica para la prevención de diabetes tipo 2 en el periodo post-gestacional.

1.2.4 Valor Teórico: La justificación de la presente tesis doctoral es profundizar en el conocimiento de la diabetes gestacional y el síndrome metabólico en nuestra población para optimizar las estrategias de su prevención, explorar protocolos alternativos de seguimiento durante la gestación para mejorar los parámetros de control.

1.2.5 Utilidad Metodológica: Con esta investigación podríamos o ayudaría a crear un nuevo instrumento para la recolección o análisis en las pacientes gestantes con SM.

\subsection{Marco referencial}

\subsubsection{Antecedentes}

Fernández et al., ${ }^{8}$ en España realizó un estudio descriptivo, prospectivo y transversal titulado "Importancia de la detección de factores de riesgo para diabetes mellitus gestacional en Granada", encontrando que, los factores de riesgo con mayor prevalencia entre las gestantes con diabetes gestacional fue la edad materna mayor o igual a los 35 años $(58,0 \%)$ y la obesidad pregestacional $(18,0 \%)$ y el antecedente familiar con el $6 \%$. La prevalencia de malformación congénita, cesárea relacionada con DMG y macrosomía fue en el $2 \%$. Se concluyó que no hubo diferencias significativas entre los factores de riesgo en las gestantes con DMG y sin DMG.

Collazo et al., ${ }^{9}$ en cuba desarrollaron un estudio observacional descriptivo retrospectivo titulado "Caracterización de los factores de riesgo para Diabetes Gestacional y su influencia durante la gestación” donde se concluyó que en las gestantes con factores de riesgo para DM, que no fueron diagnosticadas, ni 
recibieron tratamiento, fueron más frecuentes las complicaciones, versus las gestantes con DM que tenían factores de riesgo, los casos de niños macrosómicos en el primer grupo fue de $18,9 \%$, mientras que en el segundo fue de solo 10,3\%.

La diabetes gestacional (DG) presenta una modificación en el metabolismo de los hidratos de carbono que se presenta durante el embarazo. ${ }^{5}$

En Chile ${ }^{10}$, los reportes oficiales han estimado una prevalencia entre 3 y $5 \%$, porcentajes que se incrementan con entre 10 a $14 \%$ en gestantes con antecedentes familiares de diabetes, edad por encima de los 30 años, el exceso de peso, diabetes gestacional presente en anteriores embarazos. Desde el análisis socioeconómico las mujeres con un bajo nivel suelen presentar una elevada incidencia de DG demás del exceso de peso antes del embarazo $(37,8 \%)^{10}$.

Masuzaki et al $^{11}$., realizó un estudio sobre los factores de riesgo donde el diagnóstico del índice de masa corporal (IMC), el peso, los antecedentes familiares de DM tipo 2 y la edad son los indicadores de mayor riesgo para SM. Con frecuencia los valores de presión arterial se elevan no siendo diagnóstico definitivo para HTA en las gestantes con DG.

Jensen et al., ${ }^{12}$ en su investigación "en pacientes gestantes con antecedentes de glicemia elevada antes de salir gestando" encontraron que presentaron hiperglicemia en sus controles durante su embarazo. La predisposición al tener SM en mujeres con hiperglicemia previa a la gestación es 10 veces mayor si tuvo como antecedente exceso de peso pregestacional. Concluyendo que la edad es el factor predictor sobre todo en mujeres jóvenes adultas el diagnóstico de SM.

Isomaa et al., ${ }^{13}$ estudiaron "la asociación del SM con resistencia a la insulina, tolerancia anormal a la glucosa, dislipidemias, obesidad e hipertensión arterial, en 
mujeres"; participaron 21 mujeres con DG y 18 mujeres sin DG. Se encontró diferencias significativas con relación a la insulinorresistencia y variables asociadas al SM como "glicemia, triglicéridos, HTA e IMC. Las gestantes con DG presentaron las variables asociadas al SM, siendo la principal la resistencia a la insulina".

Tiikkainen et al., ${ }^{14}$ investigó la "comparación de las variables asociadas a la resistencia insulínica en gestantes con DG y sin DG"; los cuales identificaron que los factores asociados al SM son determinantes para presentar DG. Confirmando que la DG es una etapa previa al diagnóstico definitivo del SM.

Intriago et al., ${ }^{15}$ en Ecuador realizó una investigación con el objetivo en “determinar la influencia de la alimentación en los trastornos metabólicos que complican el embarazo en mujeres de 20 a 40 años.” se encontró que el 35\% de las gestantes tuvo antecedentes familiares de DM, el 42\% antecedentes de HTA, un $15 \%$ con antecedentes obesidad. Respecto al consumo de alimentos, el $43 \%$ solo come 2 veces al día, con predominio de alimentos grasos en el 36\% y un 55\% desconoce la alimentación en la gestación, antecedentes de DM (14\%) y con HTA $(38 \%)$

La obesidad en la gestación causa una dificultad para la salud pública, al incrementar los riesgos obstétricos y neonatales con posible mortalidad materna e infantil. Datos oficiales epidemiológicos en nuestro país ha reportado que el sobrepeso en la mujer en edad fértil se ha duplicado en los últimos años, así también las gestantes con exceso de peso. El exceso de peso está vinculado con diversas complicaciones maternas como la HTA, DG, aborto espontáneo, parto pretérmino, muerte fetal intrauterina, macrosomía fetal y mayores casos de cesáreas. $^{2}$ 
Lozano et al.,16 en su investigación titulada "sobrepeso y obesidad en el embarazo: complicaciones y manejo" con el objetivo en "describir las complicaciones maternas asociadas al sobrepeso y obesidad durante el embarazo e identificar los datos más adecuados sobre una vida saludable y prevención de sobrepeso y obesidad en la mujer embarazada." Realizó una extensa revisión bibliográfica en bases de salud como "Google Académico, Medline, Pubmed, Cochrane y Scielo" durante el periodo 2010 al 2016. Concluyendo que la DG y la HTA son las principales dificultades asociadas al exceso de peso en la gestante.

La condición nutricional previa y durante la gestación es determinante para la prevención de riesgos de mortalidad materna, crecimiento y desarrollo del feto, la mortalidad intrauterina, el tiempo gestacional y las complicaciones asociadas del parto. $^{17}$

La obesidad es de alto riesgo durante la gestación, lo que implica la presencia de aborto espontáneo, DG, HTA, preeclampsia, parto prematuro, alteraciones del trabajo de parto y mayor tasa de cesárea. ${ }^{18}$

Ruiz et al., ${ }^{19}$ en su investigación titulada "Síndrome metabólico en gestantes de alto riesgo obstétrico" con el objetivo en "determinar la prevalencia del síndrome metabólico (SM) y sus componentes en mujeres embarazadas con alto riesgo obstétrico". Realizó un estudio observacional, descriptivo y transversal; participaron 181 gestantes. El diagnóstico de SM se estableció según las definiciones de la IDF y NCEP ATP III excluyendo la circunferencia abdominal. Se encontró una prevalencia de SM del 49,7 \%; con dislipidemia $(80,1 \%)$; colesterol HDL bajo (60,9\%); glicemia alterada en ayunas $(19,2 \%)$, con obesidad el 75,5 \%. Además, hubo asociación estadísticamente significativa entre SM y 
preeclampsia y entre la obesidad y la preeclampsia. Se concluyó que la prevalencia de SM en gestantes con preeclampsia fue elevada y significativa.

El síndrome metabólico (SM) se asocia a un aumento significativo en el riesgo de diabetes, enfermedad cardíaca coronaria y movimiento, dando por resultado un aumento de la mortalidad cardiovascular.

Ferrada et al. ${ }^{20}$, en investigación titulada "Relación entre diabetes gestacional y síndrome metabólico" con el objetivo en determinar la relación entre la diabetes gestacional y el desarrollo de síndrome metabólico al final del período puerperal. Realizó un estudio de casos y controles en el Hospital de Curanilahue, en el sur de Chile, incluyendo 58 mujeres con diabetes gestacional durante su embarazo, en el día 42 del periodo puerperal (grupo de estudio) y 58 mujeres puerperales del mismo hospital, que había un fisiológico embarazo (grupo control). Se midieron los triglicéridos, colesterol HDL, niveles de glucosa en ayuno y post prandial sangre, presión arterial, circunferencia de la cintura, peso y altura. El síndrome metabólico fue diagnosticado según los criterios de la National colesterol Education Program (NCEP - ATP III). Se obtuvo que el peso promedio, índice de masa corporal, circunferencia de la cintura, la glucemia y triglicéridos fueron significativamente mayores en el grupo de estudio y de colesterol HDL fue significativamente menor en el grupo control. Estos resultados sugieren una relación significativa entre la diabetes gestacional y la aparición del síndrome metabólico.

Por otro lado, hay estudios que sugieren que las mujeres con SM en el primer trimestre de la gestación suelen presentar mayor riesgo para desarrollar diabetes mellitus gestacional. ${ }^{21}$ 
Akinci et al., ${ }^{22}$ con el objetivo en "determinar los factores predictores del posterior desarrollo del SM en mujeres con una diabetes mellitus gestacional previa", encontraron que la prevalencia del SM es elevada en esta población y que los factores predictores fueron la obesidad pregestacional, la ganancia de peso y la resistencia a la insulina.

Yogev et al., ${ }^{23}$ en su estudio concluyeron que la DMG está asociada a tener un niño y que éste tiene mayor riesgo de obesidad precoz, diabetes tipo 2 durante la adolescencia y desarrollo del SM.

Todos los trabajos descritos, y alguno más sugieren, pues, que hay una significativa relación entre la diabetes gestacional y la ocurrencia de $\mathrm{SM} .{ }^{20}$

\subsubsection{Marco teórico}

\subsubsection{Definición de diabetes gestacional}

La diabetes gestacional "se caracteriza por la hiperglucemia, es decir, el aumento del azúcar en la sangre; que aparece durante la gestación y suele presentar cifras elevadas a los parámetros normales, sin embargo, no para diagnosticas DM". ${ }^{21}$

Las mujeres con diabetes gestacional corren "mayor riesgo de sufrir complicaciones durante el embarazo y el parto. Además, tanto ellas como sus hijos corren mayor riesgo de padecer diabetes de tipo 2 en el futuro". ${ }^{2}$

\subsubsection{Fisiopatología de la diabetes gestacional}

En la gestación se presentan variaciones en el metabolismo materno, esto con la finalidad de crear un entorno para la embriogénesis, el crecimiento del feto y la maduración. Además, la presencia de reservas nutricionales que promueva las demandas en los siguientes meses de la gestación y hasta después de éste. Un embarazo normal se caracteriza por el aumento de los valores de glucosa postprandiales y la resistencia a la insulina. ${ }^{23}$ 
En la DG, los principales mecanismos son: la resistencia a la insulina y el deterioro en la función de las células beta. Diversos estudios así lo demuestran; sumado a ello está el IMC elevado, índice de masa corporal, la obesidad central, e hiperlipidemia, entre otras características. ${ }^{25}$

Las hormonas placentarias son las que generan la resistencia a la insulina, mediante algunas hormonas como "lactógeno humano placentario, progesterona, cortisol, hormona del crecimiento y prolactina"; cuyo rol hormonal en la gestación es aumentar el tamaño de la placenta, pero en la DMG limita el metabolismo de la gestante luego del parto. ${ }^{26} \mathrm{Al}$ progresar la gestación, la resistencia a la insulina aumenta. ${ }^{26}$

Diversas variables influyen en la alteración de la glucosa a nivel bioquímico, "la actividad de la tirosina cinasa, la cual es responsable de la fosforilación de sustratos celulares, disminución de la expresión del receptor de insulina sustrato, una proteína citosólica que se une fosforilada a los sustratos intracelulares y trasmite señales y disminución de la expresión de la proteína de transporte de glucosa GLUT4 en el tejido adiposo." 25

De manera adicional, "se observa que el embarazo está marcado, en la zona del páncreas, por hipertrofia de células $\mathrm{B}$ e hiperplasia, con el fin de compensar la disminución de la insulina, la sensibilidad a ésta y el aumento de las necesidades de insulina". 26

La producción de glucosa hepática en ayunas aumenta $30 \%$ a medida que avanza el embarazo; también se incrementa de manera importante el tejido adiposo, con mayor predominio en las gestantes con obesidad, lo que resulta en aumento de las demandas en insulina.

En las células de los tejidos diana (sistema musculoesquelético y hepático, primordialmente) se han descrito defectos posreceptor en la cascada de señales, desencadenada por la insulina, lo que favorece la intolerancia a la glucosa o resistencia insulínica; esto en mujeres con factores de riesgo.

Hay otro proceso fisiopatológico relacionado con la evolución de la diabetes mellitus gestacional es que estas pacientes, principalmente las que 
tienen obesidad, resultarán con una respuesta inflamatoria persistente como consecuencia de las citocinas proinflamatorias tipo factor de necrosis tumoral (TNF) e interleucina 6 (IL-6), que a su vez inducen resistencia insulínica. La resistencia a la insulina y el daño en la función de las células beta son los principales mecanismos participantes en la generación de la diabetes mellitus gestacional. ${ }^{27}$

\subsubsection{Definición de síndrome metabólico}

Se denomina Síndrome Metabólico (SM) “al conjunto de alteraciones metabólicas y cardiovasculares que están relacionadas con la resistencia a la insulina y la obesidad abdominal." 28

La resistencia a la insulina (RI) tiene una gran importancia "en el desarrollo del SM, hasta tal punto que se afirma que el SM es la expresión clínica de la RI. La RI es la disminución de la sensibilidad a la insulina en la captación y metabolismo de la glucosa en los tejidos periféricos (fundamentalmente tejido graso y músculo), lo que produce, en consecuencia, aumento de la síntesis y secreción de la insulina por las células $\beta$ del páncreas.”

La RI tiene efectos adversos:

- "Proliferación de las células espumosas y depósito de lípidos en la pared vascular".

- "Aumento de la tensión arterial"

- “Aumento de la producción del factor plasminógeno activador inhibidor tipo 1, fibrinógeno, factor VII y de proteína C Reactiva."

- "Aumento de la lipólisis en el tejido adiposo. En efecto, la RI aumenta la actividad de la lipasa, enzima responsable de la lipólisis en el adipocito, produciendo aumento de los ácidos grasos libres". 
- “Alteraciones en el metabolismo lipoproteico, ya que la lipasa hepática (que aumenta con la RI), produce aumento de los triglicéridos y del LDL, colesterol y disminución del HDL.”

- "Aumento de peso por deterioro en la termogénesis."

Por todo lo antes mencionado, la RI incrementa el riesgo cardiovascular y la presencia de diabetes tipo $2 .^{28}$

Teniendo en cuenta que la obesidad abdominal, es el primer filtro para diagnosticar el SM. ${ }^{29}$

El SM se “diagnostica con la presencia de más de 3 de los siguientes criterios según lo establece la ATP III ${ }^{29}$ (National Cholesterol Education Program Adult Treatment Panel III)" (tabla 1).

Tabla 1: Criterios para el diagnóstico del síndrome metabólico según ATP-III, $2001 .^{29}$

\begin{tabular}{|c|c|c|}
\hline Criterios & Hombres & Mujeres \\
\hline Obesidad abdominal & $>102 \mathrm{~cm}$ & $>88 \mathrm{~cm}$ \\
\hline Triglicéridos & $\geq 150 \mathrm{mg} / \mathrm{dl}$ & $\geq 150 \mathrm{mg} / \mathrm{dl}$ \\
\hline cHDL & $<40 \mathrm{mg} / \mathrm{dl}$ & $<50 \mathrm{mg} / \mathrm{dl}$ \\
\hline Presión arterial & $\geq 130 / \geq 85 \mathrm{mmHg}$ & $\geq 130 / \geq 85 \mathrm{mmHg}$ \\
\hline Glucemia en ayunas & $\geq 110 \mathrm{mg} / \mathrm{dl}$ & $\geq 110 \mathrm{mg} / \mathrm{dl}$ \\
\hline
\end{tabular}

\section{El síndrome metabólico y el embarazo}

Es pertinente el abordaje de este síndrome, dada la prevalencia de enfermedades crónicas no transmisibles en obesidad, DM e HTA y su consiguiente influencia sobre la salud reproductiva de la población. ${ }^{30}$ 
La gestación condiciona a un síndrome metabólico temporal con una disfunción epitelial. En la gestación normal el gasto cardiaco se incrementa propio del estado fisiológico, y después de la semana 20, resistencia a la insulina y dislipemia.

Todos estos factores son proaterogénicos y se encuentran en mayor prevalencia en la gestante que desarrolla HTA. Este SM continua luego del parto y se evidencia con un IMC elevado, HTA y triglicéridos elevados y disminución del colesterol HDL. ${ }^{31}$

En ese sentido la gestación genera un entorno muy similar al del SM, hasta tal punto que, en mujeres con SM o algunos de sus componentes, el embarazo, per se, puede empeorar la hiperglucemia, dislipemia y de la HTA. ${ }^{31}$

Por otro lado, en mujeres con criterio del SM previas a la gestación como obesidad, HTA, DM, dislipemia, tienen un elevado riesgo de disfunción placentaria que puede conllevar a la muerte del feto.

Por lo tanto, la investigación basada en evidencia en la predicción de modificar el perfil metabólico de la mujer antes de salir embarazada, como la restricción calórica o un incremento del nivel de actividad física, puede lograr disminuir en un futuro el riesgo de disfunción placentaria. ${ }^{32}$

\subsubsection{Síndrome metabólico y diabetes gestacional}

La DMG perjudica entre un 6 a $8 \%$ de las gestantes asociándose con un aumento de la morbimortalidad fetal y neonatal. ${ }^{33}$

Con presencia de RI e hiperinsulinemia, esta última es una irregularidad inicial en la diabetes mellitus tipo 2, con un incremento de la producción hepática de glucosa, que se traduce posteriormente en hiperglucemia en la gestante. ${ }^{33}$ 
Y esta RI esta beneficiada por la secreción placentaria de hormonas diabetógenas como "la hormona del crecimiento, cortisol, lactógeno placentario, progesterona, así como por el aumento de la adiposidad materna". ${ }^{33}$

En las mujeres con diabetes mellitus gestacional tienen:

- Un mayor riesgo para presentar diabetes luego del parto con cifras desde el 6 al $70 \%$, en función del tiempo transcurrido y los factores de riesgo existentes.

- Tres veces mayor riesgo de presentar SM.

- Los hijos tendrán un riesgo de 8 veces más de presentar diabetes durante la adultez joven, así como una mayor prevalencia de sobrepeso y obesidad.

- Además, un mayor riesgo en los hijos de mujeres con DMG se ha asociado tanto con la macrosomía como con el bajo peso al nacimiento.

Como consecuencia, la DMG conlleva a un círculo vicioso que aumenta el desarrollo de diabetes en las futuras generaciones. ${ }^{34}$

\subsection{Objetivos e hipótesis}

\subsubsection{Objetivo general}

- Determinar la relación entre la diabetes gestacional y el síndrome metabólico en el Instituto Materno Perinatal, Lima, 2018.

\subsubsection{Objetivos específicos}

- Conocer las características sociodemográficas de las gestantes Instituto Materno Perinatal, Lima, 2018.

- Conocer la prevalencia de la diabetes gestacional en el Instituto Materno Perinatal, Lima, 2018.

- Determinar la prevalencia del síndrome metabólico en las gestantes en el Instituto Materno Perinatal, Lima, 2018. 
- Identificar los criterios para desarrollar el síndrome metabólico en las gestantes en el Instituto Materno Perinatal, Lima, 2018.

- Determinar el desarrollo del síndrome metabólico en las gestantes con diabetes en el Instituto Materno Perinatal, Lima, 2018.

\subsubsection{Hipótesis}

$\mathrm{H}_{1}$ : Existe relación significativa entre la diabetes gestacional y el síndrome metabólico en el Instituto Materno Perinatal, Lima, 2018.

$\mathrm{H}_{0}$ : No existe relación significativa entre la diabetes gestacional y el síndrome metabólico en el Instituto Materno Perinatal, Lima, 2018. 


\section{CAPÍTULO II}

\subsection{Tipo y diseño de investigación}

Se realizó un estudio observacional, analítico de corte transversal, tipo caso-control.

\begin{tabular}{|c|c|c|c|}
\hline $\begin{array}{c}\text { Factor de riesgo } \\
\text { o protección }\end{array}$ & Casos & Controles & \\
\hline Expuestos & $\mathrm{a}$ & $\mathrm{b}$ & $\mathrm{a}+\mathrm{b}$ \\
\hline No expuestos & $\mathrm{c}$ & $\mathrm{d}$ & $\mathrm{c}+\mathrm{d}$ \\
\hline & $\mathrm{a}+\mathrm{c}$ & $\mathrm{b}+\mathrm{d}$ & $\mathrm{a}+\mathrm{b}+\mathrm{c}+\mathrm{d}$ \\
\hline
\end{tabular}

- Proporción de casos expuestos: a / $(\mathrm{a}+\mathrm{c})$

- Proporción de controles expuestos: $b /(b+d)$

- Razón de prevalencia: (a x d) / (c x b)

\subsection{Población y muestra}

La población son las 14,134 gestantes atendidas en el Instituto Materno Perinatal, durante el año 2017 en el consultorio de nutrición.

Para el tamaño de muestra se realizó un muestreo probabilístico. Se utilizó la fórmula para la población finita, es decir conocemos el total de la población gestante y se calculó la muestra: 


$$
n=\frac{N^{*} Z_{a}^{2} p^{*} q}{d^{2} *(N-1)+Z_{a}^{2} * p^{*} q}
$$

donde:

- $\quad \mathrm{N}=$ Total de la población

- $\quad \mathrm{Za} 2=1.962($ si la seguridad es del 95\%)

- $\mathrm{p}=$ proporción esperada (en este caso $5 \%=0.05)$

- $\mathrm{q}=1-\mathrm{p}($ en este caso $1-0.05=0.95)$

- $\quad \mathrm{d}=$ precisión (en este caso deseamos un 3\%).

Se obtuvo una muestra de 412 gestantes. Luego mediante el muestreo aleatorio simple se seleccionaron los casos y los controles.

De la muestra inicial se seleccionaron dos grupos: pacientes con diagnóstico de diabetes gestacional y control glucémico en el último año (casos) y una muestra aleatoria de pacientes embarazadas sin diagnóstico de diabetes gestacional en el curso de su embarazo del año 2017 (controles).

Teniendo 206 gestantes para los casos y 206 gestantes para los controles.

\subsection{Criterios de selección}

\section{A. Criterios de inclusión:}

- Gestantes que se atendieron en el lugar de estudio.

- Gestantes que desearon participar y firmaron voluntariamente el consentimiento informado.

- Gestantes con diagnóstico de diabetes gestacional. 


\section{B. Criterios de exclusión:}

- Gestantes con enfermedades crónicas transmisibles

- Gestaciones con embarazos múltiples

- Gestantes sin diagnóstico de diabetes gestacional.

- Gestantes sin síndrome metabólico

- Gestantes adolescentes.

- Gestantes que se atendieron en el lugar de estudio.

- Gestantes que desearon participar y firmaron voluntariamente el consentimiento informado. 


\subsection{Operacionalización de variables}

\begin{tabular}{|c|c|c|c|c|c|c|c|}
\hline VARIABLE & $\begin{array}{c}\text { DEFICION } \\
\text { CONCEPTUAL }\end{array}$ & $\begin{array}{l}\text { DIMENSION } \\
\text { OPERACIONAL }\end{array}$ & DIMENSIONES & $\begin{array}{c}\text { TIPO DE } \\
\text { VARIABLE }\end{array}$ & $\begin{array}{c}\text { ESCALA } \\
\text { DE } \\
\text { MEDICION }\end{array}$ & INDICADORES & INSTRUMENTO \\
\hline IMC Pregestacional & $\begin{array}{l}\text { "Índice de masa } \\
\text { corporal que está } \\
\text { determinada por el } \\
\text { peso y talla del } \\
\text { paciente." }\end{array}$ & 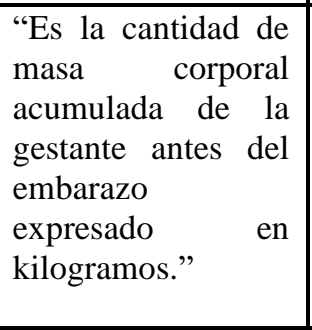 & $\begin{array}{l}\text { Bajo peso } \\
\text { Normal } \\
\text { Sobrepeso } \\
\text { Obesidad }\end{array}$ & Cualitativa & Ordinal & $\begin{array}{l}\text { Bajo peso }<18.5 \\
\mathrm{Kg} / \mathrm{m}^{2}: 1 \\
\text { Normal:18.5 a } 25 \\
\mathrm{~kg} / \mathrm{m}^{2}: 2 \\
\text { Sobrepeso: } \geq 25 \\
\mathrm{~kg} / \mathrm{m}^{2}: 3 \\
\text { Obesidad I: } \geq 30 \mathrm{~kg} \\
/ \mathrm{m}^{2}: 4\end{array}$ & Historia nutricional \\
\hline $\begin{array}{c}\text { Perímetro } \\
\text { abdominal } \\
\text { pregestacional }\end{array}$ & $\begin{array}{l}\text { "Es la medición de la } \\
\text { distancia alrededor } \\
\text { del abdomen en un } \\
\text { punto específico, por } \\
\text { lo general a nivel del } \\
\text { ombligo. } \\
\text { medición" Esta }\end{array}$ & 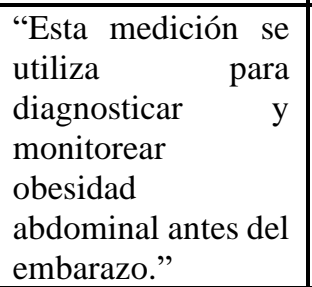 & $\begin{array}{l}\text { Con Obesidad } \\
\text { abdominal } \\
\text { Sin Obesidad } \\
\text { abdominal }\end{array}$ & Cualitativa & Nominal & $\begin{array}{l}>88 \mathrm{~cm} \text { con obesidad } \\
\text { abdominal: } 1 \\
<88 \mathrm{~cm} \text { sin obesidad } \\
\text { abdominal: } 2\end{array}$ & Historia nutricional \\
\hline $\begin{array}{l}\text { IMC según edad } \\
\text { gestacional }\end{array}$ & $\begin{array}{l}\text { "Índice de masa } \\
\text { corporal que está } \\
\text { determinada por el } \\
\text { peso y talla del } \\
\text { paciente." }\end{array}$ & $\begin{array}{l}\text { "Índice de masa } \\
\text { corporal que está } \\
\text { determinada por el } \\
\text { peso y talla en la } \\
\text { gestante." }\end{array}$ & $\begin{array}{l}\text { Bajo peso } \\
\text { Normal } \\
\text { Sobrepeso } \\
\text { Obesidad }\end{array}$ & Cualitativa & Ordinal & $\begin{array}{l}\text { Bajo peso: } 1 \\
\text { Normal: } 2 \\
\text { Sobrepeso: } 3 \\
\text { Obesidad: } 4\end{array}$ & Historia nutricional \\
\hline $\begin{array}{c}\text { Síndrome } \\
\text { metabólico ATP III }\end{array}$ & $\begin{array}{l}\text { "National } \\
\text { Cholesterol } \\
\text { Education Program- } \\
\text { Adult Treatment } \\
\text { Panel III) elaborado } \\
\text { National Cholesterol } \\
\text { Education Program } \\
\text { (NCEP)" }\end{array}$ & $\begin{array}{l}\text { "Diagnóstico del } \\
\text { síndrome } \\
\text { metabólico, } \\
\text { considerando como } \\
\text { mínimo tres } \\
\text { factores de riesgo." }\end{array}$ & $\begin{array}{l}\text { Obesidad } \\
\text { abdominal: } \\
\text { circunferencia de } \\
\text { cintura }>102 \mathrm{~cm} \text { en } \\
\text { varones y }>88 \mathrm{~cm} \\
\text { en mujeres). Para la } \\
\text { gestante será } \\
\text { pregestacional. }\end{array}$ & Cualitativa & Nominal & $\begin{array}{l}\text { Presencia de síndrome } \\
\text { metabólico: } 1 \\
\text { Ausencia de síndrome } \\
\text { metabólico: } 2\end{array}$ & Historia nutricional \\
\hline
\end{tabular}




\begin{tabular}{|c|c|c|c|c|c|c|c|}
\hline & & & $\begin{array}{l}\text { Hipertrigliceridemia } \\
>150 \mathrm{mg} / \mathrm{dl} \\
\text { cHDL bajo }(<40 \\
\mathrm{mg} / \mathrm{dl} \text { en varones; < } \\
50 \mathrm{mg} / \mathrm{dl} \text { en mujeres } \\
\mathrm{HTA}>130 / 85 \\
\mathrm{mmHg} \\
\text { Glucemia en ayunas } \\
\text { elevada > } 110 \\
\mathrm{mg} / \mathrm{dl} .\end{array}$ & & & & \\
\hline $\begin{array}{c}\text { Colesterol HDL } \\
\text { bajo }\end{array}$ & $\begin{array}{lr}\text { "Lipoproteínas } & \text { que } \\
\text { transportan } & \text { el } \\
\text { colesterol desde los } \\
\text { tejidos del cuerpo } \\
\text { hasta el hígado." }\end{array}$ & $\begin{array}{lr}\text { "relacionado } & \text { con } \\
\text { los riesgos } & \text { de } \\
\text { enfermedades } & \\
\text { cardíacas } & \text { o } \\
\text { coronarias". } & \end{array}$ & $\begin{array}{l}\text { Bajo } \\
\text { normal }\end{array}$ & Cualitativa & Ordinal & $\begin{array}{l}<40 \text { Colesterol } \mathrm{HDL} \\
\text { bajo: } 1 \\
>40 \text { Colesterol } \\
\text { bajo: } 2\end{array}$ & Historia nutricional \\
\hline $\begin{array}{l}\text { Hipertensión } \\
\text { arterial }\end{array}$ & $\begin{array}{l}\text { "Es la elevación } \\
\text { persistente de la } \\
\text { presión arterial por } \\
\text { encima de los } \\
\text { valores establecidos } \\
\text { como normales." }\end{array}$ & $\begin{array}{l}\text { "Es la elevación } \\
\text { persistente de la } \\
\text { presión arterial por } \\
\text { encima de los } \\
\text { valores } \\
\text { establecidos como } \\
\text { normales durante la } \\
\text { gestación:" }\end{array}$ & $\begin{array}{l}\text { Elevado } \\
\text { Normal }\end{array}$ & Cualitativa & Ordinal & $\begin{array}{l}\text { HTA > 130/85 mmHg: } \\
1 \\
\text { HTA < } 130 / 85 \mathrm{mmH} ; 2\end{array}$ & Tarjeta perinatal \\
\hline Diabetes mellitus & $\begin{array}{l}\text { "La glucosa en } \\
\text { sangre es una prueba } \\
\text { que mide la cantidad } \\
\text { de azúcar (glucosa) } \\
\text { en una muestra de } \\
\text { sangre." }\end{array}$ & $\begin{array}{l}\text { "La glucosa en } \\
\text { sangre es una } \\
\text { prueba que mide la } \\
\text { cantidad de azúcar } \\
\text { (glucosa) en una } \\
\text { muestra de sangre } \\
\text { en la gestación." }\end{array}$ & $\begin{array}{l}\text { Normal } \\
\text { Diabetes }\end{array}$ & Cualitativa & Ordinal & $\begin{array}{l}\text { Glucemia en ayunas } \\
\text { elevada }>110 \mathrm{mg} / \mathrm{dl}: 1 \\
\text { Glucemia en ayunas } \\
\text { elevada < } 110 \mathrm{mg} / \mathrm{dl}: 2\end{array}$ & Tarjeta perinatal \\
\hline
\end{tabular}




\begin{tabular}{|c|c|c|c|c|c|c|c|}
\hline Hipertrigliceridemia & 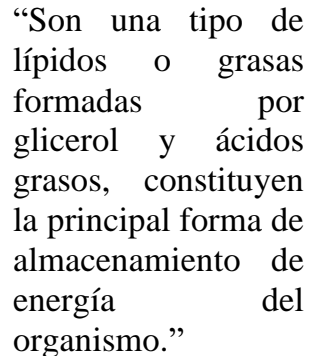 & $\begin{array}{l}\text { "Es una prueba que } \\
\text { mide la cantidad de } \\
\text { el exceso de } \\
\text { concentración } \\
\text { sérica } \\
\text { triglicérido en una } \\
\text { muestra de sangre." }\end{array}$ & $\begin{array}{l}\text { Normal } \\
\text { Hipertrigliceridemia }\end{array}$ & Cualitativa & Ordinal & $\begin{array}{l}\text { Normal: }<150 \mathrm{mg} / \mathrm{dl}: 1 \\
\text { Elevado: }>150 \mathrm{mg} / \mathrm{dl}: 2\end{array}$ & Tarjeta perinatal \\
\hline
\end{tabular}




\subsection{Instrumento}

Se diseñó un instrumento, ficha de recolección de datos, con preguntas relacionadas en su primera parte con datos sociodemográficos, luego los datos de la valoración nutricional (peso, talla, perímetro abdominal), datos bioquímicos: triglicéridos, HDL colesterol y glucemia en ayunas. (anexo 2).

El instrumento fue sometido a validez de contenido mediante juicio de expertos, considerándose para ello ocho profesionales en nutrición, los resultados obtenidos fueron procesados a través de la prueba binomial (anexo 3). Y siguiendo las recomendaciones de los jueces expertos se realizó una prueba piloto para determinar la validez del instrumento mediante la prueba coeficiente de correlación de Pearson (anexo 4) y la confiabilidad estadística mediante la prueba Kuder Richardson (anexo $5)$.

\subsection{Técnicas y procedimientos de recolección}

Antes de recopilar la información se procedió con los trámites administrativos para la aprobación del presente protocolo de investigación por el Comité de ética de la Universidad San Ignacio de Loyola y el Comité de ética, investigación y docencia del Instituto Materno Perinatal.

Durante los meses de febrero a marzo del 2018 se visitó el Instituto Materno Perinatal en el horario de 8:00 a.m. a 1:00 p.m. para recoger la información según la ficha de recolección de datos, previa explicación del estudio y las gestantes procedieron a firmar el consentimiento informado (anexo 1).

Posterior a la recolección de la información, los datos fueron ingresados a una matriz según su codificación en la operacionalización de variables. Luego procesados en el programa SPSS versión 22.0. 


\subsection{Análisis de datos}

A continuación, se describen las etapas del análisis de datos realizados en la presente investigación:

\subsubsection{Estadística descriptiva:}

Para las variables sociodemográficas (edad, grado de instrucción, ocupación y estado civil). Para tiempo gestacional, aplicando medidas de tendencia central para la variable cuantitativa y tabla de frecuencias para las variables cualitativas.

\subsubsection{Estadistica inferencial}

a) Evaluacion para analisis bivariante de las variables del estudio con Chi cuadrado

b) Estimacion medicion de asociaion, evaluar la magnitud de la asociacion, evaluar la fuerza de asociacion entre el factor de estudio y evento se utilizo el rango de prevalencia, indicando el rango de exposicion entre casos y control.

c) Estimación del error estándar para proceder a efectuar inferencia a tráves del intervalo de confianza.

\subsection{Consideraciones éticas}

Se contó con la aprobación del Comité de investigación del Instituto Materno Perinatal y el Comité de investigación de la Universidad San Ignacio de Loyola. Se explicó la finalidad de la investigación a las gestantes, respetando su autonomía, y las participantes que aceptaron voluntariamente firmaron el consentimiento informado (anexo 1). 


\section{CAPÍTULO III}

\subsection{Presentación de resultados}

\begin{tabular}{|c|c|c|c|c|c|c|}
\hline \multicolumn{7}{|c|}{$\begin{array}{l}\text { Tabla 1: Datos sociodemográficos de la gestante en el Instituto Nacional Materno } \\
\text { Perinatal, Lima, } 2018 .\end{array}$} \\
\hline \multirow{2}{*}{$\begin{array}{c}\text { Características de las } \\
\text { Gestantes }\end{array}$} & \multicolumn{2}{|c|}{$\begin{array}{c}\text { Casos } \\
n=206\end{array}$} & \multicolumn{2}{|c|}{$\begin{array}{c}\text { Controles } \\
n=206\end{array}$} & \multirow[t]{2}{*}{$\mathbf{R P} *$} & \multirow[t]{2}{*}{ IC 95\% } \\
\hline & $\mathbf{n}$ & $\%$ & $\mathbf{n}$ & $\%$ & & \\
\hline \multicolumn{7}{|l|}{ Edad } \\
\hline $20-35$ años & 183 & 88,8 & 192 & 93,2 & 0,54 & $1,08-1,98$ \\
\hline $36-42$ años & 23 & 11,2 & 14 & 6,8 & 0,49 & $0,12-0,49$ \\
\hline \multicolumn{7}{|l|}{ Grado de instrucción } \\
\hline Primaria Completa & 36 & 17,5 & 39 & 18,9 & 0,41 & $0,41-0,81$ \\
\hline Secundaria Completa & 147 & 71,4 & 152 & 73,8 & 0,53 & $0,29-0,72$ \\
\hline Superior Completa & 05 & 2,4 & 02 & 1,0 & 0,47 & $0,65-1,09$ \\
\hline Técnica Completa & 18 & 8,8 & 13 & 6,3 & 0,67 & $0,22-0,45$ \\
\hline \multicolumn{7}{|l|}{ Ocupación } \\
\hline Ama de casa & 169 & 82,0 & 178 & 86,4 & 0,73 & $0,34-0,79$ \\
\hline Empleada & 12 & 5,8 & 13 & 6,3 & 0,39 & $0,26-0,33$ \\
\hline Estudiante & 09 & 4,4 & 06 & 2,9 & 0,58 & $0,89-1,21$ \\
\hline Independiente & 16 & 7,8 & 09 & 4,4 & 0,41 & $0,29-0,45$ \\
\hline \multicolumn{7}{|l|}{ Estado Civil } \\
\hline Soltera & 29 & 14,1 & 13 & 6,3 & 0,95 & $1,01-1,34$ \\
\hline Casada & 18 & 8,7 & 15 & 7,3 & 0,67 & $1,25-1,31$ \\
\hline Conviviente & 159 & 77,2 & 178 & 86,4 & 0,91 & $1,09-1,43$ \\
\hline \multicolumn{7}{|l|}{ Tiempo gestacional } \\
\hline $20-40$ semanas & 197 & 95,6 & 198 & 96,1 & 0,32 & $1,61-3,54$ \\
\hline 41 - a más & 09 & 4,4 & 08 & 3,9 & 0,31 & $1,21-3,42$ \\
\hline
\end{tabular}

- RP: Razón de prevalencia

- IC: Intervalo de confianza

La tabla 1 muestra los resultados de los datos sociodemográficos de la población estudiada según los casos y los controles. En ambos grupos de investigación se observan características similares. 
En los casos, según el rango de edad se presenta en las gestantes en la etapa adulta joven, es decir, entre 20 y 35 años con el mayor porcentaje (88,8\%). El rango de 36 a 42 años con el 11,2\%; situación semejante se muestra en el grupo control donde las gestantes jóvenes alcanzan el 93,2\% y entre 32 a 42 años 6,8\%.

Respecto al grado de instrucción en los casos, el 71,4\% de gestantes muestra secundaria completa, seguido del $17,5 \%$ con primaria completa, el 8,8\% presenta estudios técnicos completos y sólo el 2,4\% nivel universitario completo.

En el grupo control es mayor la proporción de gestantes con secundaria completa $(73,8 \%)$, seguido del $18,9 \%$ con primaria completa, el $6,3 \%$ presenta estudios técnicos completos y sólo el 1,0\% nivel universitario completo.

Respecto a la ocupación de la gestante, en los casos el 82,0\% refiere ser ama de casa. El 7,8\% son independientes, el 5,8\% empleadas y el 4,4\% son estudiantes. Situación similar se presenta en el grupo control donde el $86,4 \%$ realiza los quehaceres del hogar, el 6,3\% son empleados, el 4,4\% son trabajadoras independientes y el 2,9\% son estudiantes.

El estado civil también se indagó, el 77,2\% refiere ser conviviente, el 14,1\% es madre soltera y el 8,7\% está casada. En los controles, esta situación es semejante; donde el $86,4 \%$ convive con su pareja, el 7,3\% está casada y el 6,3\% es madre soltera.

Los resultados de acuerdo con el tiempo de gestación muestran que el 95,6\% de gestantes se encuentran entre las 20 a 40 semanas, condición similares en los controles con el 96,1\%. De 41 a más semanas el $4,4 \%$ en los casos y 3,9\% en los controles, respectivamente. 


\begin{tabular}{|c|c|c|c|c|c|c|}
\hline \multicolumn{7}{|c|}{$\begin{array}{l}\text { Tabla 2: Valoración antropométrica, bioquímica y clínica en la gestante en el Instituto Nacional } \\
\text { Materno Perinatal, Lima, 2018. }\end{array}$} \\
\hline Características & Media & DS & Mediana & Min & Max & $\mathbf{p}$ \\
\hline \multicolumn{7}{|l|}{\begin{tabular}{|l} 
VALORACIÓN ANTROPOMETRICA \\
\end{tabular}} \\
\hline IMC pregestacional $\left(\mathrm{kg} / \mathrm{cm}^{2}\right)$ & 26,7 & 4,36 & 28,9 & 16,6 & 37,1 & 0,991 \\
\hline IMC gestacional $\left(\mathrm{kg} / \mathrm{cm}^{2}\right)$ & 31,2 & 4,23 & 31,5 & 22,2 & 39,9 & 0,995 \\
\hline Ganancia de peso para la edad gestacional & 10,6 & 4,15 & 11,0 & 2,3 & 24 & 0,000 \\
\hline Circunferencia abdominal pregestacional $(\mathrm{cm})$ & 105,3 & 132,2 & 81,0 & 68 & 99 & 0,998 \\
\hline \multicolumn{7}{|l|}{ VALORACIÓN BIOQUÍMICA } \\
\hline Triglicéridos (mg/dl) & 97,3 & 40,7 & 78,0 & 70 & 196 & 0,999 \\
\hline Colesterol HDL (mg/dl) & 47,9 & 5,7 & 50,0 & 32 & 57 & 0,997 \\
\hline Glicemia (mg/dl) & 100,6 & 30,4 & 91,0 & 61 & 191 & 0,999 \\
\hline \multicolumn{7}{|l|}{ VALORACIÓN CLINICA } \\
\hline PA Sistólica (mmHg) & 125,6 & 62,6 & 130,0 & 90 & 130 & 0,999 \\
\hline PA Diastólica (mmHg) & 80,5 & 11,3 & 85,0 & 55 & 96 & 0,998 \\
\hline
\end{tabular}

La tabla 2 muestra las características antropométricas, bioquímica y clínica de la gestante, el IMC pregestacional es 26,7 y el IMC gestacional 31,2. La ganancia de peso para la edad gestacional es significativa p: 0.000 con una media de 10,6. La circunferencia pregestacional con una media de 105,3. Los triglicéridos (97.3 mg/dl), el colesterol HDL (47,9 mg/dl) y la glicemia (100,6 mg/dl.). La presión arterial obtuvo una media de 125,6/80,5 $\mathrm{mmHg}$.

\begin{tabular}{|c|c|c|c|c|c|c|}
\hline \multirow[t]{2}{*}{ Criterios } & \multicolumn{2}{|c|}{$\begin{array}{c}\text { Casos } \\
\mathrm{n}=\mathbf{2 0 6} \\
\end{array}$} & \multicolumn{2}{|c|}{$\begin{array}{l}\text { Control } \\
\mathrm{n}=\mathbf{2 0 6} \\
\end{array}$} & \multirow[t]{2}{*}{$\mathbf{X}^{2 *}$} & \multirow[t]{2}{*}{ Valor $\mathbf{p}$} \\
\hline & $\mathbf{n}$ & $\%$ & $\mathbf{n}$ & $\%$ & & \\
\hline Hipertigliceridemia & 84 & 40,7 & 01 & 0,5 & 5,43 & 0,021 \\
\hline Obesidad abdominal pregestacional & 178 & 86,4 & 16 & 7,8 & 6,32 & 0,000 \\
\hline Colesterol HDL bajo & 51 & 24,7 & 0 & 0 & 14,5 & 0,002 \\
\hline HTA* & 197 & 95,6 & 16 & 7,8 & 21,2 & 0,000 \\
\hline Diabetes mellitus & 142 & 68,9 & 16 & 7,8 & 11,0 & 0,004 \\
\hline
\end{tabular}

*HTA: Hipertensión arterial

La Tabla 3 muestra los criterios del síndrome metabólico según la definición de ATP III, en los casos la mayor prevalencia se presentó con HTA $(95,6 \%)$, seguido de la obesidad 
abdominal pregestacional con el $86,4 \%$, la diabetes mellitus en el $68,9 \%$ y la hipertrigliceridemia con el 40,7\%. El 24,7\% de los estudiados presentó el colesterol HDL bajo.

En el grupo control se observa que el $7,8 \%$ con HTA, diabetes mellitus y obesidad abdominal pregestacional, respectivamente. Sólo el $0.5 \%$ presentó hipertrigliceridemia. Los valores del colesterol HDL se encuentran dentro de los parámetros normales según la definición.

Tabla 4: Prevalencia del síndrome metabólico en las gestantes en el Instituto Nacional Materno Perinatal, Lima, 2018.

\begin{tabular}{|c|c|c|c|c|c|c|c|}
\hline \multirow{2}{*}{$\begin{array}{c}\text { Síndrome } \\
\text { Metabólico } \\
\text { (ATP III) }\end{array}$} & \multicolumn{2}{|c|}{$\begin{array}{c}\text { Casos } \\
\mathbf{n = 2 0 6}\end{array}$} & \multicolumn{2}{c|}{$\begin{array}{c}\text { Control } \\
\mathbf{n = 2 0 6}\end{array}$} & $\mathbf{R P}^{*}$ & $\mathbf{X}^{\mathbf{2} * *}$ & Valor $\mathbf{p}$ \\
\cline { 2 - 6 } & $\mathbf{n}$ & $\mathbf{\%}$ & $\mathbf{n}$ & $\mathbf{\%}$ & \multirow{2}{*}{1,12} & \multirow{2}{*}{15,2} & 0,104 \\
\hline SM: SI & 206 & 100 & 16 & 7,8 & & \\
\hline SM: NO & - & - & 190 & 92,2 & & & \\
\hline
\end{tabular}

*RP: Razón de prevalencia

**Prueba Chi-cuadrado

La Tabla 4 muestra la prevalencia del síndrome metabólico en la población estudiada, este diagnóstico se presentó en la totalidad de los casos (100\%), es decir la combinación de tres o más criterios como la obesidad abdominal, diabetes mellitus e hipertrigliceridemia e HTA. Sólo el 7,8\% en los controles, con un RP: 1,12.

Tabla 5: Relación entre la diabetes gestacional y el síndrome metabólico en el Instituto Nacional Materno Perinatal, Lima, 2018.

\begin{tabular}{|c|c|c|c|c|c|c|}
\hline \multirow{4}{*}{$\begin{array}{c}\text { Diabetes } \\
\text { gestacional }\end{array}$} & \multicolumn{2}{|c|}{ Síndrome metabólico en la gestante } & \multirow{2}{*}{\begin{tabular}{c} 
RP* \\
Casos \\
\cline { 2 - 5 }
\end{tabular}} & \multicolumn{2}{|c|}{$\begin{array}{c}\text { Controles } \\
\mathbf{n = 2 0 6}\end{array}$} & \multirow{2}{*}{$\mathbf{p}$} \\
\cline { 2 - 5 } & $\mathbf{n}$ & $\mathbf{\%}$ & $\mathbf{n}$ & $\mathbf{\%}$ & & \\
\cline { 2 - 5 } & 142 & 68,9 & 16 & 7,8 & 7,69 & 0,000 \\
\hline
\end{tabular}

*RP: Razón de prevalencia 
La Tabla 5 muestra la relación del síndrome metabólico en la población estudiada, este diagnóstico se presentó en un 68,9\% de los casos y un 7,8\% en los controles con un RP: 7,69 y una significancia de 0,000 .

El estudio no presento sesgo de selección porque se tomaron en cuenta los criterios de inclusión tomando como base los datos de la historia clínica y tarjeta perinatal, así mismo no tuvo sesgo del recuerdo en cuanto como para el peso pre natal y perímetro abdominal porque fueron incluidas en el estudio de investigación pacientes del primer trimestre, donde luego para la realización de la investigación a las 20 semanas. 


\subsection{Discusión}

Las diferencias de los promedios de peso materno, el IMC pregestacional y obesidad abdominal encontrados, coinciden con los hallazgos de Fernández et al., ${ }^{8}$ y Collazo et al. ${ }^{9}$, quienes reconocen al sobrepeso y la obesidad como factores predictores para el riesgo vascular y mortalidad materna; además reportan el incremento de la obesidad abdominal como un factor clave de la insulinoresistencia.

La frecuencia elevada y significativa, de la hipertensión en las gestantes de este estudio, concuerda con el estado de insulinoresistencia durante el embarazo, cifras similares a lo reportado por Masuzaki et al ${ }^{11}$, y Jensen et al., ${ }^{12}$; pero mayores a Isomaa et al., ${ }^{13}$.

Con relación a los criterios del síndrome metabólico según la definición de ATP III, en los casos la mayor prevalencia se presentó con HTA $(95,6 \%)$, seguido de la obesidad abdominal pregestacional con el $86,4 \%$, la diabetes mellitus en el $68,9 \%$ y la hipertrigliceridemia con el 40,7\%. Los valores del colesterol HDL se encuentran dentro de los parámetros normales según la definición.

Tiikkainen et al., ${ }^{14}$ e Intriago et al., ${ }^{15}$ estudiaron mujeres con DG comparándolas con pacientes sin DG, presentando un perfil de riesgo materno que consistió en glicemia ayunas y post prandial, triglicéridos, colesterol HDL, presión arterial e IMC, concluyeron que las pacientes con DG un riesgo materno aumentado para SM, lo que coincide con los resultados de este estudio.

La prevalencia del síndrome metabólico en la población estudiada, este diagnóstico se presentó en la totalidad de los casos (100\%), es decir la combinación de tres o más criterios como la obesidad abdominal, diabetes mellitus e hipertrigliceridemia e HTA. Sólo el 7,8\% en los controles, con un RP: 1,12. La proporción de pacientes con SM y con DG observadas en este estudio, concuerda con la prevalencia señalada por la OMS en que 
la DG se considera precursora de la diabetes mellitus tipo 2 (DM2), según lo señalado por Ferrada et al. ${ }^{20}$ y Akinci et al., ${ }^{22}$ y cifras por debajo según la investigación de Yogev et al., ${ }^{23}$.

La relación del síndrome metabólico en la población estudiada, este diagnóstico se presentó en un 68,9\% de los casos y un 7,8\% en los controles con un RP:7.69 y una significancia de 0,000 .

Los resultados obtenidos de niveles séricos de triglicéridos, glicemia, en el grupo de estudio, concuerdan con niveles requeridos para realizar el diagnóstico de SM, siendo posible determinar que es aproximadamente 8 veces más probable $(\mathrm{RP}=7,69)$, que una paciente que presenta DG desarrolle también SM al término del puerperio, comparada con pacientes que no presentan DG (grupo control). Esto permite concluir que existe una fuerte relación entre diabetes gestacional y síndrome metabólico.

Los resultados obtenidos nos permiten reflexionar sobre el rol de la DG en la patogénesis del SM, ya que la conjugación de los factores de riesgo a los que está expuesta la embarazada con DG las deja en una situación de riesgo y vulnerabilidad, necesaria de atender en futuras investigaciones.

Por último, destacamos la importancia de realizar en todas las mujeres con diabetes gestacional, y con mayor énfasis en las gestantes con SM el seguimiento, de manera de detectar precozmente el desarrollo de diabetes, e intervenir en la disminución de su riesgo global. 


\section{CONCLUSIONES}

- Existe una relación significativa entre la diabetes gestacional y el síndrome metabólico en la gestante con un RP: 7.69 y una significancia de 0,000. siendo que las gestantes con diabetes gestacional aumentan 8 veces el riesgo de padecer síndrome metabólico.

- Las características sociodemográficas de las gestantes la mayor prevalencia de según la edad de 20 a 35 años para los casos fue $88.8 \%$ y en control 93.2\%, el grado de instrucción es secundaria con $71.4 \%$ en casos y control $73.8 \%$, en ocupación fue amas de casa con $82 \%$ en los casos y $77.2 \%$ en control y de acuerdo al tiempo gestacional de 20 a 40 semanas fue $95,6 \%$ casos y $96.1 \%$ control.

- La prevalencia de la diabetes gestacional en los casos fue el 68,9\% y el 7,8\% en los controles.

- la prevalencia del síndrome metabólico en las gestantes fue el 100\% en los casos y el $7,8 \%$ en los controles.

- De acuerdo con los criterios del síndrome metabólico en los casos la mayor prevalencia se presentó con HTA $(95,6 \%)$, seguido de la obesidad abdominal pregestacional con el $86,4 \%$, la diabetes mellitus en el $68,9 \%$ y la hipertrigliceridemia con el 40,7\%. en el grupo control se observa el $24,7 \%$ de los estudiados presentó el colesterol HDL bajo, el 7,8\% con HTA, diabetes mellitus y obesidad abdominal pregestacional, respectivamente. 


\section{RECOMENDACIONES}

- La prevención en el sobrepeso y/o la obesidad es fundamental para disminuir la prevalencia de la diabetes de tipo 2, la consejería nutricional y el fomento de estilos de vida saludables deberían abordarse frente a esta problemática.

- El plan nutricional durante el embarazo que cursa con diabetes debe orientarse a mejorar o mantener un adecuado estado nutricional, optimizar el control glucémico y lipídico, evitar la hiperglucemia materna prepandial y reducir las excursiones de la glucemia postprandial.

- Para reducir el riesgo de complicaciones maternas y fetales es necesario que la atención esté a cargo de un equipo interdisciplinario de profesionales sanitarios.

- Efectuar un seguimiento de peso con la curva de incremento de peso para embarazadas según IMC.

- La educación alimentario nutricional, toda paciente con diagnóstico de diabetes debe recibir adecuada consejería hasta lograr los objetivos metabólicos.

- Fomentar el consumo de alimentos sanos y reducir la ingesta de alimentos malsanos y bebidas azucaradas.

- Realizar futuras investigaciones acerca de los factores de riesgo y la prevención en la diabetes gestacional. 


\section{REFERENCIAS BIBLIOGRÁFICAS}

1. Organización Mundial de la Salud. OMS. Disponible en: https://www.who.int/es/news-room/fact-sheets/detail/diabetes

2. González-Ruiz MN, Rodríguez-Bandala C, Salcedo Vargas M, Martínez-Lara E y col. Actualidades en diabetes gestacional. Rev Sanid Milit Mex 2014; $68: 276-282$

3. WHO Mortality Database [base de datos en línea]. Ginebra, Organización Mundial de la Salud. 2016. Disponible en: http://apps. who.int/healthinfo/statistics/mortality/causeofdeath_query

4. American Diabetes Association. Strategies for improving care sec 1. In: Standards of medical care in diabetes 2015. Diabetes Care 2015; 38:55-57.

5. Schiavone M, Putoto G, Laterza F, Pizzol D. Gestational diabetes: an overview with attention for developing countries. Endocr Regul 2016; 50:62-71

6. Castillo-Cartín A. Diabetes mellitus gestacional. Generalidades. Rev Med Costa Rica y Centroamérica 2011; 58:109-113.

7. Merzouk H, Madani S, Boualga A. Age-related changes in cholesterol metabolism in macrosomic offspring of rats with streptozotocin-induced diabetes. J Lipid Res, 42 .2001, pp. 1152-9.

8. Fernández C, Luna M, Lorenzo M. Importancia de la detección de factores de riesgo para diabetes mellitus gestacional. Granada (España), Enero - junio 2016. Index Enferm vol.25 no.1-2 
9. Collazo M, Gordon C, Álvarez S. Caracterización de los factores de riesgo para Diabetes Gestacional y su influencia durante la gestación. Mediciego 2013 Cuba; 19 (Supl. 2) pag. 1

10. CEDIP Guías clínicas perinatales 2005; 16: 219-23.

11. Masuzaki H, Paterson J. A transgenic model of visceral obesity and the metabolic syndrome. Science 2001; 294: 2166-70.

12. Jensen DM, Sorensen B, Feilberg-Jorgensen N, Westergaard JG, Becknielsen H. Maternal and perinatal outcomes in 143 Danish women with gestational diabetes mellitus and 143 controls with a similar risk profile. Diabet Med 2000; 17: 281-6.

13. Isomaa $\mathrm{B}$, Henricsson $\mathrm{M}$. The metabolic syndrome influences the risk of chronic complications in patients with II diabetes. Diabetologia 2001; 44: 1148-54.

14. Tiikkainen M, Marjo T, Annamaija H, et al. Liver-fat accumulation and insulin resistance in obese women with previous gestational diabetes. Obesity Research 2002; 10: 859-67.

15. Dom. Cien., ISSN: 2477-8818 Vol. 3, núm. 4, julio, 2017, pp. 462-475 Trastornos metabólicos que complican el embarazo http://dx.doi.org/10.23857/dom.cien.pocaip.2017.3.4.jul. 462-475 Disponible: URL:http://dominiodelasciencias.com/ojs/index.php/es/index

16. Sobrepeso y Obesidad en el Embarazo: Complicaciones y Manejo. Alejandra Lozano Bustillo, Waleska Rosario Betancourth Melendez, Linda José Turcios Urbina , José Emanuel Cueva Nuñez, Daniela Michell Ocampo Eguigurems, Cinthia Vanessa Portillo Pineda. Archivos de Medicina ISSN 1698-9465 2016 Vol. 12 No. 3: 11 doi: $10.3823 / 1310$ 
17. Barrera C. Obesidad y Embarazo. Revista Médica Clínica La Condes 23: 2012.154-158.

18. Moreno M. Definición Y Clasificación De La Obesidad. Rev med clin condes 23: 2012. 124-128.

19. Rosario Ruiz,1 J. Max Gonzales-Gallegos2 y Eunice MirandaNavia3Síndrome metabólico en gestantes de alto riesgo obstétricoRev Soc Peru Med Interna 2014; vol 27 (3)

20. Ferrada Cecilia, Molina Marta, Cid Luis, Riedel Gisela, Ferrada Cristina, Arévalo Rodrigo. Relación entre diabetes gestacional y síndrome metabólico. Rev. méd. Chile [Internet]. 2007 Dic [citado 2018 Dic 05] ; 135 (12): 1539-1545. Disponible en: https://scielo.conicyt.cl/scielo.php?script=sci_arttext\&pid=S0034$\underline{98872007001200006 \& \operatorname{lng}=\mathrm{es}}$

21. L. Chatzi, E. Plana, A. Pappas, D Alegkakis, P Karakosta, V Daraki

The metabolic syndrome in early pregnancy and risk of gestational diabetes mellit us

Diabetes Metab, 35 (2009), pp. 490-494 http://dx.doi.org/10.1016/j.diabet.2009.07.0 03

22. B. Akinci, A. Celtik, S. Yener, S. Yesil. Prediction of developing metabolic sy ndrome after gestational diabetes mellitus.2013.

23. Y. Yogev, G.H. Visser. Obesity, gestational diabetes and pregnancy outcome Semin Fetal Neonatal Med, 14. 2009. pp. 77-84 http://dx.doi.org/10.1016/j.sin y.2008.09.002

24. Ley SH, Hamdy, O, Mohan V, Hu FB. Prevention and management of type 2 diabetes: dietary components and nutritional strategies. Lancet. 2014;383(9933):1999-2007. 
25. Nolan C, Damm P, Prentki Ml. Type 2 diabetes across generations: from pathophysiology to prevention and management. Lancet. 2011;378(9786):169-181.

26. Vickers MH. Early life nutrition, epigenetics and programming of later life disease. Nutrients. 2014;6:(6)2165-217

27. . Diagnostic criteria and classification of hyperglycaemia first detected in pregnancy (WHO/NMH/ MND/13.2). Ginebra: Organización Mundial de la Salud; 2013.

28. Régimen alimentario, nutrición y prevención de enfermedades crónicas: informe de una consulta mixta FAO/OMS de expertos. OMS, Serie de Informes Técnicos 916. Ginebra, Organización Mundial de la Salud, 2003. http://www.who.int/nutrition/publications/obesity/WHO_TRS_916/es/

29. Executive Summary of The Third Report of The National Cholesterol Education Program (NCEP) Expert Panel on Detectio.n, Evaluatio.n, And Treatment of High Blood Cholesterol In Adults (Adult Treatment Panel III). JAMA. 2001; 285:2486-97.

30. Harding JL, Shaw JE, Peeters A, Guiver T, Davidson S, Magliano DJ. Mortality trends among people with type 1 and type 2 diabetes in Australia: 1997-2010. Diabetes Care. 2014;37:(9)2579-2586.

31. Noncommunicable diseases progress monitor 2015. Ginebra, Organización Mundial de la Salud, 2015.

32. Informe mundial sobre la diabetes. OMS 2016 . http://apps.who.int/iris/bitstream/handle/10665/254649/9789243565255spa.pdf;jsessionid=A91477508B2411B239E3F05DC3F4D237? sequence $=1$ 
33. Johnsson, IW, Haglund B, Ahlsson F, Gustafsson J. A high birth weight is associated with increased risk of type 2 diabetes and obesity. Pediatric Obesity. 2015;10(2):77-83.

34. Wong E, Backholer K, Gearon E, Harding J, Freak-Poli R, Stevenson C, et al. Diabetes and risk of physical disability in adults: a systematic review and meta-analysis. Lancet Diabetes Endocrinology. 2013;1:(2)106-114. 
ANEXOS 


\section{Anexo 1 \\ CONSENTIMIENTO INFORMADO}

- GESTANTES -

Institución

Investigadora

Título
: Universidad San Ignacio de Loyola

: Mg. Cecibel Guisela Aparcana Espinoza

: RELACIÓN ENTRE LA DIABETES GESTACIONAL Y EL SINDROME METABOLICO EN EL INSTITUTO NACIONAL PERINATAL, LIMA, 2018.

\section{Propósito del Estudio:}

Lo (a) estoy invitando a participar en un estudio llamado: Relación de la diabetes gestacional y el síndrome metabólico en el Instituto Nacional Materno Perinatal 2018.

Este es un estudio desarrollado por la Mg. Cecibel Guisela Aparcana Espinoza, nutricionista de formación, para obtener el grado académico de doctora en Nutrición con el objetivo en determinar La relación de la diabetes gestacional y el síndrome metabólico en el Instituto Nacional Materno Perinatal, Lima, 2018.

El síndrome metabólico, conjunción de varias enfermedades que aparecen de forma simultánea en un individuo, causados por la combinación de factores genéticos y ambientales asociados al estilo de vida como: obesidad, hipertensión, diabetes, colesterol y triglicéridos elevados. El reconocer los factores de riesgo del síndrome metabólico en la gestante sus implicancias durante el embarazo y será útil para la Salud Pública para tomar acciones en el manejo adecuado de este síndrome, así como su prevención en los controles prenatales por el equipo multidisciplinario.

\section{Procedimientos:}

Si usted acepta participar en este estudio se le harán los siguientes procedimientos:

1. Se utilizará una ficha nutricional de recolección de datos donde se le harán diversas preguntas sobre sus antecedentes personales y familiares de enfermedades crónicas., tipo de comida que consume habitualmente.

2. También se realizará la valoración nutricional y diagnostico nutricional con una balanza, tallímetro, cáliper y cinta métrica.

3. Se realizará el recojo de los datos de la tarjeta de control prenatal e historia clínica, de indicadores antropométricos prenatales, gestacionales y bioquímicos, lo que ayudará a determinar el diagnostico de síndrome metabólico.

4. Finalmente se realizará le recojo de información de la historia clínica a la ficha nutricional sobre los datos antropométricos del recién nacido para el diagnóstico nutricional. 


\section{Riesgos:}

No se prevén riesgos por participar en esta fase del estudio.

\section{Beneficios:}

Usted se beneficiará de una evaluación nutricional para el despistaje de esta enfermedad. Se le informará de manera personal y confidencial los resultados que se obtengan de las valoraciones realizadas. Los costos de todos los exámenes serán cubiertos por el estudio y no le ocasionarán gasto alguno.

\section{Costos e incentivos:}

Usted no deberá pagar nada por participar en el estudio. Igualmente, no recibirá ningún incentivo económico ni de otra índole, únicamente la satisfacción de colaborar a un mejor entendimiento y conocimiento de la enfermedad.

\section{Confidencialidad:}

Nosotros guardaremos su información con códigos y no con nombres. Si los resultados de este seguimiento son publicados, no se mostrará ninguna información que permita la identificación de las personas que participan en este estudio. Sus archivos no serán mostrados a ninguna persona ajena al estudio sin su consentimiento

\section{Derechos del paciente:}

Si usted decide participar en el estudio, puede retirarse de éste en cualquier momento, o no participar en una parte del estudio sin perjuicio alguno. Si tiene alguna duda adicional, por favor pregunte al personal del estudio, o llamar a la Lic. Cecibel Aparcana al 965-618299.

\section{CONSENTIMIENTO}

Acepto voluntariamente participar en este estudio, comprendo que cosas me van a pasar si participo en el proyecto, también entiendo que puedo decidir no participar y que puedo retirarme del estudio en cualquier momento.

Participante

Nombre:

DNI:

Fecha:

Investigadora

Nombre:

DNI:

Fecha: 


\section{FICHA DE RECOLECCIÓN DE DATOS}

"RELACIÓN ENTRE LA DIABETES GESTACIONAL Y EL SÍNDROME METABOLICO EN EL INSTITUTO MATERNO PERINATAL, LIMA, 2018”

I. Datos generales de la gestante:

1.1 Edad:

1.2 Tiempo gestacional:

1.3 Grado de instrucción:
A. Analfabeta
B. Primaria:
C. Secundaria $\square$
D. Superior

1.4 Ocupación:
A. Ama de casa
B. Independiente $\square$
C. Empleada
D. Estudiante

1.5 Estado Civil:
A. Soltera $\square$
B. Casada $\square$
C. Divorciada
D. Conviviente $\square$

II. Valoración nutricional de la gestante

2.1 Peso: $\mathrm{Kg}$

2.2 Talla: $\mathrm{cm}$.

2.3 IMC pregestacional:

2.4 Perímetro abdominal: $\mathrm{Kg} / \mathrm{m}^{2}$

2.5 IMC según edad gestacional: $\mathrm{cm}$. $\mathrm{Kg} / \mathrm{m}^{2}$

III. Datos bioquímicos en la gestante

3.1 Fecha de análisis:

3.2 Glucosa: $\mathrm{mg} / \mathrm{dl}$.

3.3 Triglicéridos: $\mathrm{mg} / \mathrm{dl}$

3.4 Colesterol HDL bajo: $\mathrm{mg} / \mathrm{dl}$

3.5 Presión arterial: $\mathrm{mmHg}$.

IV. Diagnóstico de diabetes gestacional:
4.1 Si $\square$
$4.2 \mathrm{No} \square$

V. Diagnóstico de síndrome metabólico:

$5.1 \mathrm{Si}$

$5.2 \mathrm{No} \square$ 
Anexo 3

PRUEBA BINOMIAL

GRADO DE CONCORDANCIA ENTRE LOS JUECES EXPERTOS

\begin{tabular}{|c|c|c|c|c|c|c|c|c|c|}
\hline \multirow{2}{*}{ PREGUNTAS } & \multicolumn{7}{|c|}{$\mathbf{N}^{\circ}$ DE JUECES EXPERTOS } & \multirow{2}{*}{ P } \\
\cline { 2 - 10 } & $\mathbf{1}$ & $\mathbf{2}$ & $\mathbf{3}$ & $\mathbf{4}$ & $\mathbf{5}$ & $\mathbf{6}$ & $\mathbf{7}$ & $\mathbf{8}$ & $\mathbf{1}$ \\
\hline 1 & 1 & 1 & 1 & 1 & 1 & 1 & 1 & 1 & 0.002 \\
\hline 2 & 1 & 1 & 1 & 1 & 1 & 1 & 1 & 1 & 0.002 \\
\hline 3 & 1 & 1 & 1 & 1 & 1 & 1 & 1 & 1 & 0.002 \\
\hline 4 & 1 & 1 & 1 & 1 & 1 & 1 & 1 & 1 & 0.002 \\
\hline 5 & 1 & 1 & 1 & 1 & 1 & 1 & 1 & 1 & 0.002 \\
\hline 6 & 1 & 1 & 1 & 1 & 1 & 1 & 1 & 1 & 0.002 \\
\hline 7 & 1 & 1 & 1 & 1 & 1 & 1 & 1 & 1 & 0.020 \\
\hline 8 & 1 & 1 & 1 & 1 & 1 & 1 & 1 & 1 & 0.002 \\
\hline 9 & 1 & 1 & 1 & 1 & 1 & 1 & 1 & 1 & 0.002 \\
\hline 10 & 1 & 1 & 1 & 1 & 1 & 0 & 1 & 1 & 0.020 \\
\hline 11 & 1 & 0 & 1 & 1 & 1 & 1 & 1 & 1 & 0.002 \\
\hline 12 & 1 & 0 & 1 & 1 & 1 & 1 & 1 & 1 & 0.002 \\
\hline 13 & 1 & 1 & 1 & 1 & 1 & 1 & 1 & 1 & 0.002 \\
\hline 14 & 1 & 1 & 1 & 1 & 1 & 1 & 1 & 1 & 0.002 \\
\hline 15 & 1 & 1 & 1 & 1 & 1 & 1 & 1 & 1 & 0.002 \\
\hline 16 & 1 & 1 & 1 & 1 & 1 & 1 & 1 & 1 & 0.002 \\
\hline 17 & 1 & 1 & 1 & 1 & 1 & 1 & 1 & 1 & 0.002 \\
\hline
\end{tabular}

En estos ítems se tomaron en consideración las observaciones realizadas para la elaboración del instrumento final.

$\mathrm{SI}=1$

$\mathrm{NO}=0$

Si $\mathrm{p}<0.05$ la concordancia es significativa. De acuerdo con los resultados obtenidos por cada juez experto, son menores de 0.05 , por lo tanto, el grado de concordancia es significativo. 
Anexo 4

VALIDEZ DEL INSTRUMENTO

COEFICIENTE DE CORRELACIÓN R DE PEARSON

\begin{tabular}{|c|c|c|c|}
\hline ITEM & $\begin{array}{l}\text { CORRELACIÓN } \\
\text { R DE PEARSON }\end{array}$ & ITEM & $\begin{array}{l}\text { CORRELACIÓN } \\
\text { R DE PEARSON }\end{array}$ \\
\hline $\mathbf{1}$ & 0.27 & $\mathbf{1 0}$ & 0.28 \\
\hline $\mathbf{2}$ & 0.28 & $\mathbf{1 1}$ & 0.26 \\
\hline $\mathbf{3}$ & 0.26 & $\mathbf{1 2}$ & 0.51 \\
\hline $\mathbf{4}$ & 0.25 & $\mathbf{1 3}$ & 0.42 \\
\hline $\mathbf{5}$ & 0.29 & $\mathbf{1 4}$ & 0.32 \\
\hline $\mathbf{6}$ & 0.28 & $\mathbf{1 5}$ & 0.28 \\
\hline $\mathbf{7}$ & 0.26 & $\mathbf{1 6}$ & 0.29 \\
\hline $\mathbf{8}$ & 0.25 & $\mathbf{1 7}$ & 0.29 \\
\hline $\mathbf{9}$ & 0.27 & & \\
\hline & & & \\
\hline
\end{tabular}

Cómo $\mathrm{r}>0.20$, el instrumento es válido en cada una de las preguntas. 
Anexo 5

\section{CONFIABILIDAD DEL INSTRUMENTO COEFICIENTE DE KUDER RICHARDSON}

La confiabilidad del instrumento estructura será determinada mediante el Coeficiente de “Kuder Richardson” (k).

$$
r=\left[\frac{N}{N-1}\right] X\left[1-\frac{\sum S_{i}^{2}}{S_{t}^{2}}\right]
$$

\section{Donde:}

r: Coeficiente de confiabilidad Kuder Richarson

$\mathrm{N}$ : Número de ítems

$S_{i}^{2}$ : Varianza de cada ítem

$S_{t}^{2}$ : Varianza total

Obteniéndose los siguientes resultados:

$$
\begin{gathered}
r=\left[\frac{17}{17-1}\right] \times\left[1-\frac{2,05}{5,98}\right] \\
r=\left[\frac{17}{16}\right] \times[1-0,28] \\
r=1,06 \times 0,72 \\
r=\mathbf{0 , 7 6}
\end{gathered}
$$

Se obtuvo que $r$ es 0,76 ; siendo $r>0,50$ es significativo; por lo tanto, el instrumento es confiable. 
